\title{
Aplicação de medidas de gravidade e de inconsistência de fala em crianças com transtorno fonológico
}

\author{
The use of severity measures and speech inconsistency in children \\ with speech sound disorders
}

\author{
Haydée Fiszbein Wertzner', Karina Pereira', Thaís Zemlickas Silva', Luciana de Oliveira Pagan-Neves ${ }^{1}$
}

\begin{abstract}
RESUMO
Objetivo: Descrever os índices PCC-R, IRS, IRO e IRD, PDI e IIF em crianças com transtorno fonológico, com e sem os processos fonológicos de ensurdecimento, e verificar a eficiência desses índices na identificação de diferenças entre as crianças. Métodos: Trata-se de pesquisa retrospectiva e transversal com 20 crianças com transtorno fonológico e idade entre 5 e 8 anos. Foram realizados dois agrupamentos, de acordo com a presença dos processos fonológicos de ensurdecimento de plosivas e de fricativas. Todos foram submetidos ao teste de fonologia $\mathrm{ABFW}$, no qual foi verificada a produtividade dos processos fonológicos, o número de diferentes tipos de processos fonológicos e os índices PDI, PCC-R, IRS, IRO e IRD. A prova de inconsistência de fala foi aplicada para calcular o índice de inconsistência de fala. Os dados foram submetidos à análise estatística inferencial. Resultados: $\mathrm{O}$ estudo indicou que crianças com transtorno fonológico e presença de ensurdecimento de fricativas e/ou plosivas têm maior comprometimento de fala e apresentam valores mais altos do índice de inconsistência de fala, PDI, IRS, IRO e IRD. O erro articulatório mais ocorrente em ambos os grupos foi a substituição, sendo que a distorção ocorreu mais no grupo sem os processos fonológicos de ensurdecimento. Conclusão: Os índices aplicados foram eficientes para diferenciar crianças com e sem a presença dos processos fonológicos de ensurdecimento. Há evidências de que as crianças que apresentam os processos fonológicos de ensurdecimento têm dificuldade na representação fonológica.
\end{abstract}

Descritores: Fonoaudiologia; Criança; Avaliação; Transtornos da articulação; Índice de gravidade de doença; Testes de linguagem

\begin{abstract}
Purpose: To describe the severity indexes PCC-R, IRS, IRO, IRD, PDI and SII in children with speech sound disorder with and without the phonological process of devoicing and verify its efficiency in identifying differences among children. Methods: This is retrospective and cross-sectional study involving 20 children with speech sound disorder between 5 and 8 years-old. Two subgroups were created according to the presence of the phonological process of stops and fricatives devoicing. Phonology test from the infantile language test (ABFW) was used to calculate productivity of phonological processes, the number of different types of phonological processes and the indexes PDI, PCC-R, IRS, IRO and IRD. Speech inconsistency test was applied to calculate the speech inconsistency index. Data were statistically analyzed. Results: The study demonstrated that children with speech sound disorder and the presence of fricative and/or stops devoicing were more severe and presented higher values of the speech inconsistency index, PDI, IRS, IRO and IRD. The articulatory error most observed for both groups was the substitution while distortion occurred more in the group of children without the phonological process of devoicing. Conclusion: The severity indexes used were efficient to differentiate children with from children without the presence of the phonological processes of devoicing. There is evidence that children who have the phonological process of devoicing have difficulty with the phonological representation of the sound.
\end{abstract}

Keywords: Speech, Language and Hearing Sciences; Child; Evaluation; Articulation disorders; Severity of illness index; Language tests

Trabalho realizado no Curso de Fonoaudiologia, Faculdade de Medicina, Universidade de São Paulo - USP - São Paulo (SP), Brasil.

(1) Curso de Fonoaudiologia, Faculdade de Medicina, Universidade de São Paulo - USP - São Paulo (SP), Brasil.

Conflito de interesses: Não

Contribuição dos autores: $H F W$ pesquisador principal, elaboração da pesquisa, elaboração do cronograma, aprovação da versão final; $K P, T Z S$ e $L O P N$ responsáveis pelo levantamento da literatura, coleta e análise dos dados, redação do artigo; $L O P N$ responsável pela submissão e trâmites do artigo, correção da redação do artigo. Endereço para correspondência: Haydée Fiszbein Wertzner. R. Cipotânea, 51, Cidade Universitária, São Paulo (SP), Brasil, CEP: 05360-000.

E-mail: hfwertzn@usp.br

Recebido em: 3/5/2013; Aceito em: 12/8/2013 


\section{INTRODUÇÃO}

O fato de o Transtorno Fonológico (TF) ser uma das alterações de fala e linguagem mais ocorrentes na população infantil, torna-o alvo de investigação de diversos estudos. Apesar das características necessárias para que a criança seja diagnosticada como portadora de TF serem bastante conhecidas, os estudos atuais buscam detalhar a causa, gravidade, tipo de erro (substituição, omissão e distorção) e de processos fonológicos predominantes, bem como os subtipos do transtorno, que lhe atribuem grande heterogeneidade ${ }^{(1)}$.

OTF é identificado a partir da dificuldade em usar as regras do sistema fonológico, que pode ser identificada pela presença de processos fonológicos ${ }^{(2-4)}$.

Dentre os processos fonológicos mais ocorrentes e determinantes para caracterizar o TF em crianças estão: Ensurdecimento de Plosivas (EP), Ensurdecimento de Fricativas (EF), Frontalização de Palatal (FP), Simplificação do Encontro Consonantal (SEC) e Simplificação de Líquidas (SL) ${ }^{(5,6)}$. Identificar os processos fonológicos facilita a compreensão das regras fonológicas que a criança com TF usa de forma adequada e aquelas que ela ainda simplifica.

Um dos índices mais utilizados por autores nacionais ${ }^{(7-10)}$ e internacionais ${ }^{(1)}$ é o que mede a Porcentagem de Consoantes Corretas - Revisada (PCC-R) ${ }^{(11)}$ que considera como erros somente as substituições e as omissões e avalia as distorções como acertos. O Process Density Index (PDI) ${ }^{(12,13)}$ é um índice de gravidade do TF que se apoia na ocorrência dos PF. O seu cálculo contabiliza o número total de processos fonológicos e os divide pelo número total de palavras analisadas na amostra.

Um aspecto bastante descrito no TF são os tipos de erros encontrados na fala das crianças. A utilização de índices que quantifiquem os tipos de erros detectados na avaliação auxilia nas comparações entre crianças e também são úteis para o acompanhamento terapêutico ${ }^{(9)}$. Na literatura, são descritos alguns índices e, entre eles, os índices relativos ${ }^{(14)}$, que são calculados a partir da divisão do número de erros específicos de omissão (IRO), distorção (IRD), ou substituição (IRS), pelo número total de erros na amostra de fala e que permitem verificar o tipo de erro mais ocorrente dentre todos os tipos produzidos.

Atualmente, sabe-se da necessidade de se complementar o diagnóstico do TF com provas que verifiquem o subtipo da alteração de acordo com o processamento de fala mais alterado, ou seja: cognitivo-linguístico, motor e perceptivo-auditivo. Uma das provas utilizadas para verificar a presença de dificuldade na programação fonológica ${ }^{(15,16)}$, considerado como o processo responsável por selecionar e organizar a produção do fonema, é a prova de inconsistência de fala, que mostra o Índice de Inconsistência de Fala (IIF) ${ }^{(15)}$. Autores ${ }^{(17,18)}$ afirmam que a variabilidade na produção faz parte do desenvolvimento típico, mas pode estar presente em crianças com TF.

Assim, a partir da importância do detalhamento do diagnóstico do TF e da grande ocorrência dos processos fonológicos de ensurdecimento de plosivas e fricativas em crianças com TF, o objetivo deste estudo foi descrever os índices PCC-R, IRS, IRO e IRD, PDI e IIF em crianças com transtorno fonológico, com e sem os processos fonológicos de ensurdecimento, bem como verificar a eficiência desses índices na identificação de diferenças entre as crianças.

\section{MÉTODOS}

Esta pesquisa foi aprovada pela Comissão de Ética em Pesquisa da Faculdade da Faculdade de Medicina da Universidade de São Paulo (USP), CAPPesq (nº 192-11). Todos os responsáveis assinaram o Termo de Consentimento Livre e Esclarecido. Trata-se de pesquisa retrospectiva e transversal, em que foram analisadas as provas coletadas dos sujeitos que fazem parte do banco de dados da rotina de atendimento de um serviço de fonoaudiologia da cidade de São Paulo.

A partir dos protocolos de avaliação analisados, foram selecionadas para participar da pesquisa, 20 crianças com TF, com idade entre 5 e 8 anos, de ambos os gêneros. Para serem incluídas na pesquisa as crianças tiveram o diagnóstico de TF realizado por um serviço de fonoaudiologia da cidade de São Paulo. Os sujeitos selecionados foram agrupados em dois grupos, sendo o primeiro (G1) composto por dez crianças sem a presença dos processos fonológicos de $\mathrm{EP}$ e EF, e o segundo $(\mathrm{G} 2)$ por dez crianças com a presença desses processos fonológicos.

Os critérios de inclusão no G1 foram: ter idade entre 5 e 8 anos; apresentar alterações fonológicas na avaliação da Fonologia $^{(19)}$ do Teste de Linguagem Infantil - ABFW ${ }^{(20)}$; apresentar os processos fonológicos de EF e EP com ocorrência inferior a $25 \%$ nas duas provas de fonologia (nomeação de figuras e imitação de palavras); ter desempenho adequado para a idade nos outros campos da linguagem e não apresentar queixas de dificuldade auditiva.

Os critérios de inclusão no G2 foram: ter idade entre 5 e 8 anos; apresentar alterações fonológicas no teste de fonologia ${ }^{(19)}$ do Teste de Linguagem Infantil - $\mathrm{ABFW}^{(20)}$; apresentar os processos fonológicos de EF e EP com ocorrência superior a $25 \%$ em pelo menos uma das duas provas do teste de fonologia (nomeação de figuras e imitação de palavras); ter desempenho adequado para a idade nos outros campos da linguagem e não apresentar queixas de dificuldade auditiva.

A aplicação das provas foi gravada e filmada utilizando-se uma câmera JVC-Everio ${ }^{\circledR}$ e gravador digital ZOOM H2. Para a gravação em áudio, foi usado o microfone Sennheiser® (modelo-e817), acoplado ao gravador.

Após a seleção dos sujeitos, as provas de nomeação de figuras e de imitação de palavras do teste de fonologia ${ }^{(19)}$ foram analisadas e, com base na amostra de fala coletada, foi verificada a produtividade dos processos fonológicos, o número de diferentes tipos de processos fonológicos e o cálculo dos índices PDI, PCC-R, IRS, IRO e IRD. Para a análise dos índices, foram 
consideradas as possibilidades de ocorrência de erros e de processos fonológicos nas posições inicial, medial e final das palavras. Todas as respostas utilizadas nas análises deste estudo foram transcritas foneticamente por dois juízes fonoaudiólogos, sendo um aluno do $4^{\circ}$ ano do curso de fonoaudiologia da instituição de ensino onde o estudo foi desenvolvido e um aluno do curso de pós-graduação. Para fazer parte do estudo, a concordância entre as duas transcrições deveria ser superior a $90 \%$.

O IIF foi calculado a partir da prova de inconsistência de fala ${ }^{(15)}$ que consta da nomeação de 25 figuras, apresentadas em três sequências diferentes. Os sujeitos foram considerados como consistentes quando as três emissões do vocábulo foram feitas da mesma forma, independentemente do erro ou acerto da palavra-alvo, ou inconsistentes, quando a produção da palavra-alvo foi realizada de forma diferente em, pelo menos, uma das três apresentações. O valor do IIF foi calculado ${ }^{(15-20)}$ e os sujeitos foram classificados como consistentes (C) ou inconsistentes (I), de acordo com os seguintes valores de corte estabelecidos em pesquisas anteriores: entre 5 anos e 7 anos e 6 meses para meninas, 21,5\% e para meninos 31,9\% e acima de 7 anos e 7 meses para meninas, $14,5 \%$ e para meninos 17,6\%.

Para verificar a distribuição dos dados quanto à normalidade, foi aplicado o teste de Kolgomorov-Smirnov, que demonstrou distribuição diferente da normalidade e, por isso, foram então aplicadas análises inferenciais com testes não paramétricos.
Nas comparações da idade dos sujeitos e dos índices entre os grupos nas duas provas do teste de fonologia, foi utilizado o teste de Mann-Whitney. O teste exato de Fisher verificou a presença de associação entre a classificação da prova de inconsistência de fala nos grupos. O nível de significância adotado foi de 0,05 .

\section{RESULTADOS}

Não foi observada diferença em relação às idades na comparação entre os sujeitos do G1 e do G2 (p=0,544).

A comparação dos índices entre os grupos indicou diferença em ambas as provas de nomeação de figuras e de imitação de palavras para o PCC-R e o PDI. Houve evidências de diferença na prova de nomeação de figuras e tendência à significância na prova de imitação de palavras, na análise do IRS. Observou-se essa tendência, também para o IRD, somente na prova de nomeação de figuras. Os dados descritivos demonstraram, ainda, que as médias do IRD e do IRO foram maiores no G1, nas duas provas do teste de fonologia (Tabela 1).

A comparação intragrupo dos três índices relativos indicou diferença nas duas provas do teste de fonologia. O ranqueamento intragrupo apontou o IRS como sendo o índice de maior ocorrência e o IRO como o menos ocorrente (Tabela 2).

Não foram observadas diferenças em nenhum dos dois grupos, quando os índices estudados foram comparados entre

Tabela 1. Comparação entre G1 e G2 em relação aos índices nas provas do teste de fonologia

\begin{tabular}{|c|c|c|c|c|c|c|c|c|}
\hline & & \multicolumn{2}{|c|}{ G1 } & \multicolumn{2}{|c|}{ G2 } & \multirow{2}{*}{ U } & \multirow{2}{*}{ Z } & \multirow{2}{*}{ Valor de $p$} \\
\hline & & Média $( \pm D P)$ & Mediana & Média $( \pm D P)$ & Mediana & & & \\
\hline \multirow{2}{*}{ PCC-R } & Nomeação\# & $90,65( \pm 4,33)$ & 89,44 & $72,45( \pm 13,04)$ & 66,67 & 14 & $-2,723$ & $0,006^{*}$ \\
\hline & Imitação"\# & $91,42( \pm 3,45)$ & 91,58 & $72,74( \pm 13,75)$ & 70,55 & 15 & $-2,651$ & $0,008^{*}$ \\
\hline \multirow{2}{*}{ PDI } & Nomeação & $0,27( \pm 0,13)$ & 0,28 & $0,78( \pm 0,37)$ & 0,96 & 14 & $-2,724$ & $0,006^{\star}$ \\
\hline & Imitação & $0,24( \pm 0,12)$ & 0,23 & $0,75( \pm 0,40)$ & 0,78 & 16 & $-2,574$ & $0,010^{*}$ \\
\hline \multirow{2}{*}{ IRS } & Nomeação & $0,46( \pm 0,31)$ & 0,39 & $0,78( \pm 0,22)$ & 0,84 & 22 & $-2,12$ & $0,034^{*}$ \\
\hline & Imitação & $0,45( \pm 0,33)$ & 0,42 & $0,75( \pm 0,23)$ & 0,86 & 25 & $-1,89$ & 0,059 \\
\hline \multirow{2}{*}{ IRD } & Nomeação & $0,45( \pm 0,31)$ & 0,46 & $0,18( \pm 0,24)$ & 0,07 & 25.5 & $-1,86$ & 0,063 \\
\hline & Imitação & $0,45( \pm 0,34)$ & 0,42 & $0,20( \pm 0,25)$ & 0,09 & 29 & $-1,594$ & 0,111 \\
\hline \multirow{2}{*}{ IRO } & Nomeação & $0,10( \pm 0,15)$ & 0,06 & $0,05( \pm 0,06)$ & 0,00 & 40 & $-0,808$ & 0,419 \\
\hline & Imitação & $0,11( \pm 0,10)$ & 0,09 & $0,05( \pm 0,06)$ & 0,04 & 34 & $-1,221$ & 0,222 \\
\hline
\end{tabular}

*Valores significativos $(p<0,05)$ - Teste de Mann-Whitney

Legenda: "Nomeação = prova de nomeação de figuras; *\#Imitação = prova de imitação de palavras; PCC-R = porcentagem de consoantes corretas revisado; PDI = densidade de processos fonológicos; IRS = índice relativo de substituição; IRO = índice relativo de omissão; IRD = índice relativo de distorção; IIF = índice de inconsistência de fala

Tabela 2. Ranqueamento dos índices relativos nas provas de imitação de palavras e nomeação de figuras para G1 e G2

\begin{tabular}{|c|c|c|c|c|c|c|c|c|c|}
\hline & \multicolumn{4}{|c|}{ G1 } & \multicolumn{5}{|c|}{ G2 } \\
\hline & $x^{2}$ & $\mathrm{gl}$ & Valor de $p$ & Ranqueamento & & $x^{2}$ & $\mathrm{gl}$ & Valor de $p$ & Ranqueamento \\
\hline Nomeação & 10,158 & 2 & $0,006^{*}$ & IRS $>$ IRD $>$ IRO & Nomeação & 10,947 & 2 & $0,004^{*}$ & IRS $>$ IRD $>$ IRO \\
\hline Imitação & 7,947 & 2 & $0,019^{*}$ & IRS $>$ IRD $>$ IRO & Imitação & 9,800 & 2 & $0,007^{*}$ & IRS $>$ IRD $>$ IRO \\
\hline
\end{tabular}

*Valores significativos $(p<0,05)$ - ANOVA de Friedman

Legenda: $\mathrm{gl}$ = graus de liberdade; IRS = índice relativo de substituição; IRD = índice relativo de distorção; IRO = índice relativo de omissão 
as provas de nomeação de figuras e de imitação de palavras.

Os valores do IIF foram comparados e não indicaram diferença $(p=0,096)$ entre os grupos $\mathrm{G} 1$ e $\mathrm{G} 2$, apesar do $\mathrm{G} 2$ ter apresentado IIF médio $(25,89)$ maior que o G1 $(15,27)$.

A classificação dos sujeitos do G1 e do G2 como consistentes ou inconsistentes, realizada a partir do IIF, demonstrou tendência à diferença entre os grupos $(\mathrm{p}=0,07)$, sendo que no G1 a maior parte das crianças foi considerada consistente (90\%), enquanto que no G2 observou-se um número semelhante de crianças classificadas como consistentes (50\%) e inconsistentes (50\%).

A análise da correlação dos índices entre as duas provas do teste de fonologia pode ser observada para o G1 e G2 nas Tabelas 3 e 4, respectivamente.

No G1, (Tabela 3) foram verificadas correlações positivas para os índices PCC-R, IRS, IRO e IRD nas provas de nomeação de figuras e de imitação de palavras, IRD e PCC-R na prova de nomeação de figuras, IRD e PCC-R na prova de imitação de palavras e entre o IRD, na prova de nomeação de figuras, e o PCC-R, na prova de imitação de palavras. Foram também observadas correlações negativas entre os índices: IRS na prova de nomeação de figuras e PCC-R na prova de imitação de palavras, IRS e PCC-R na prova de imitação de palavras, IRS e IRD na prova de nomeação de figuras, IRS e IRD na prova de imitação de palavras, IRS na prova de imitação de palavras e IRD na prova de nomeação de figuras, IRS na prova de nomeação de figuras e IRD na prova de imitação de palavras e entre o IIF e o IRO, na prova de imitação de palavras. Houve tendência à correlação positiva entre os índices IRD da imitação e PCC-R da nomeação e tendência à correlação negativa para os índices IRS e PCC-R da nomeação, IRS da imitação e PCC-R da nomeação, IRS da imitação e IRO da nomeação e IRD da imitação e PCC-R da nomeação.

No G2, (Tabela 4), houve correlação positiva entre os índices PCC-R, IRS, IRO e IRD nas provas de imitação de palavras e de nomeação de figuras. As correlações negativas foram entre o IRS e PCC-R, IRS e IRD na prova de nomeação de figuras, IRS e IRD na prova de imitação de palavras, IRS na prova de imitação de palavras e IRD na prova de nomeação de figuras, IRS na prova de nomeação de figuras e IRD na prova

Tabela 3. Correlação entre os índices PCC-R, PDI e IIF no G1

\begin{tabular}{|c|c|c|c|c|c|c|c|c|c|c|}
\hline & & $\begin{array}{c}\text { PCCR } \\
\text { Nomeação }\end{array}$ & $\begin{array}{c}\text { PCCR } \\
\text { Imitação }\end{array}$ & $\begin{array}{c}\text { IRS } \\
\text { Nomeação }\end{array}$ & $\begin{array}{c}\text { IRO } \\
\text { Nomeação }\end{array}$ & $\begin{array}{c}\text { IRD } \\
\text { Nomeação }\end{array}$ & $\begin{array}{c}\text { IRS } \\
\text { Imitação }\end{array}$ & $\begin{array}{c}\text { IRO } \\
\text { Imitação }\end{array}$ & $\begin{array}{c}\text { IRD } \\
\text { Imitação }\end{array}$ & IIF \\
\hline \multirow{3}{*}{$\begin{array}{l}\text { PCC-R } \\
\text { Imitação }\end{array}$} & Rho & 0,719 & 1,000 & & & & & & & \\
\hline & Valor de $p$ & $0,010^{*}$ & . & & & & & & & \\
\hline & $\mathrm{N}$ & 10 & 10 & & & & & & & \\
\hline \multirow{3}{*}{$\begin{array}{l}\text { IRS } \\
\text { Nomeação }\end{array}$} & Rho & $-0,518$ & $-0,784$ & 1,000 & & & & & & \\
\hline & Valor de $p$ & 0,062 & $0,004^{*}$ & . & & & & & & \\
\hline & $\mathrm{N}$ & 10 & 10 & 10 & & & & & & \\
\hline \multirow{3}{*}{$\begin{array}{l}\text { IRO } \\
\text { Nomeação }\end{array}$} & Rho & $-0,242$ & 0,241 & $-0,419$ & 1,000 & & & & & \\
\hline & Valor de $p$ & 0,250 & 0,251 & 0,114 & . & & & & & \\
\hline & $\mathrm{N}$ & 10 & 10 & 10 & 10 & & & & & \\
\hline \multirow{3}{*}{$\begin{array}{l}\text { IRD } \\
\text { Nomeação }\end{array}$} & Rho & 0,591 & 0,699 & $-0,903$ & 0,088 & 1,000 & & & & \\
\hline & Valor de $p$ & $0,036^{*}$ & $0,012^{*}$ & $<0,001^{*}$ & 0,405 & . & & & & \\
\hline & $\mathrm{N}$ & 10 & 10 & 10 & 10 & 10 & & & & \\
\hline \multirow{3}{*}{$\begin{array}{l}\text { IRS } \\
\text { Imitação }\end{array}$} & Rho & $-0,494$ & $-0,784$ & 0,915 & $-0,488$ & $-0,818$ & 1,000 & & & \\
\hline & Valor de $p$ & 0,073 & $0,004^{*}$ & $<0,001^{*}$ & 0,076 & $0,002^{*}$ & . & & & \\
\hline & $\mathrm{N}$ & 10 & 10 & 10 & 10 & 10 & 10 & & & \\
\hline \multirow{3}{*}{$\begin{array}{l}\text { IRO } \\
\text { Imitação }\end{array}$} & Rho & $-0,092$ & $-0,192$ & 0,061 & 0,530 & $-0,261$ & $-0,043$ & 1,000 & & \\
\hline & Valor de $p$ & 0,400 & 0,298 & 0,434 & 0,058 & 0,233 & 0,454 & . & & \\
\hline & $\mathrm{N}$ & 10 & 10 & 10 & 10 & 10 & 10 & 10 & & \\
\hline \multirow{3}{*}{$\begin{array}{l}\text { IRD } \\
\text { Imitação }\end{array}$} & Rho & 0,471 & 0,692 & $-0,894$ & 0,307 & 0,912 & $-0,948$ & $-0,137$ & 1,000 & \\
\hline & Valor de $p$ & 0,085 & $0,013^{*}$ & $<0,001^{*}$ & 0,194 & $<0,001^{*}$ & $<0,001^{\star}$ & 0,353 & . & \\
\hline & $\mathrm{N}$ & 10 & 10 & 10 & 10 & 10 & 10 & 10 & 10 & \\
\hline \multirow{3}{*}{ IIF } & Rho & 0,262 & 0,292 & $-0,297$ & $-0,344$ & 0,333 & $-0,333$ & $-0,596$ & 0,389 & 1,000 \\
\hline & Valor de $p$ & 0,232 & 0,207 & 0,202 & 0,165 & 0,173 & 0,173 & $0,035^{\star}$ & 0,133 & . \\
\hline & $\mathrm{N}$ & 10 & 10 & 10 & 10 & 10 & 10 & 10 & 10 & 10 \\
\hline
\end{tabular}

*Valores significativos $(p<0,05)$ - Coeficiente de Correlação de Spearman

Legenda: $\mathrm{N}=$ nomeação; $\mathrm{PCC}-\mathrm{R}$ = porcentagem de consoantes corretas revisado; PDI = Process Density Index; IRS = índice relativo de substituição; IRO = índice relativo de omissão; IRD = índice relativo de distorção; IIF = índice de inconsistência de fala 
Tabela 4. Correlação entre os índices PCC-R, PDI e IIF no G2

\begin{tabular}{|c|c|c|c|c|c|c|c|c|c|c|}
\hline & & $\begin{array}{c}\text { PCCR } \\
\text { Nomeação }\end{array}$ & $\begin{array}{c}\text { PCCR } \\
\text { Imitação }\end{array}$ & $\begin{array}{c}\text { IRS } \\
\text { Nomeação }\end{array}$ & $\begin{array}{c}\text { IRO } \\
\text { Nomeação }\end{array}$ & $\begin{array}{c}\text { IRD } \\
\text { Nomeação }\end{array}$ & $\begin{array}{c}\text { IRS } \\
\text { Imitação }\end{array}$ & $\begin{array}{c}\text { IRO } \\
\text { Imitação }\end{array}$ & $\begin{array}{c}\text { IRD } \\
\text { Imitação }\end{array}$ & IIF \\
\hline \multirow{3}{*}{$\begin{array}{l}\text { PCC-R } \\
\text { Imitação }\end{array}$} & Rho & 0,875 & 1,000 & & & & & & & \\
\hline & Valor de $p$ & $<0,001^{*}$ & . & & & & & & & \\
\hline & $\mathrm{N}$ & 10 & 10 & & & & & & & \\
\hline \multirow{3}{*}{$\begin{array}{l}\text { IRS } \\
\text { Nomeação }\end{array}$} & Rho & $-0,578$ & $-0,488$ & 1,000 & & & & & & \\
\hline & Valor de $p$ & $0,040^{*}$ & 0,076 & . & & & & & & \\
\hline & $\mathrm{N}$ & 10 & 10 & 10 & & & & & & \\
\hline \multirow{3}{*}{$\begin{array}{l}\text { IRO } \\
\text { Nomeação }\end{array}$} & Rho & $-0,130$ & $-0,445$ & $-0,096$ & 1,000 & & & & & \\
\hline & Valor de $p$ & 0,360 & 0,099 & 0,396 & . & & & & & \\
\hline & $\mathrm{N}$ & 10 & 10 & 10 & 10 & & & & & \\
\hline \multirow{3}{*}{$\begin{array}{l}\text { IRD } \\
\text { Nomeação }\end{array}$} & Rho & 0,399 & 0,455 & $-0,905$ & $-0,249$ & 1,000 & & & & \\
\hline & Valor de $p$ & 0,127 & 0,093 & $<0,001^{*}$ & 0,244 & . & & & & \\
\hline & $\mathrm{N}$ & 10 & 10 & 10 & 10 & 10 & & & & \\
\hline \multirow{3}{*}{$\begin{array}{l}\text { IRS } \\
\text { Imitação }\end{array}$} & Rho & $-0,467$ & $-0,474$ & 0,888 & 0,178 & $-0,939$ & 1,000 & & & \\
\hline & Valor de $p$ & 0,087 & 0,083 & $<0,001^{*}$ & 0,312 & $<0,001^{*}$ & . & & & \\
\hline & $\mathrm{N}$ & 10 & 10 & 10 & 10 & 10 & 10 & & & \\
\hline \multirow{3}{*}{$\begin{array}{l}\text { IRO } \\
\text { Imitação }\end{array}$} & Rho & $-0,399$ & $-0,585$ & $-0,049$ & 0,829 & $-0,155$ & 0,141 & 1,000 & & \\
\hline & Valor de $p$ & 0,127 & $0,038^{*}$ & 0,446 & $0,001^{*}$ & 0,334 & 0,349 & . & & \\
\hline & $\mathrm{N}$ & 10 & 10 & 10 & 10 & 10 & 10 & 10 & & \\
\hline \multirow{3}{*}{$\begin{array}{l}\text { IRD } \\
\text { Imitação }\end{array}$} & Rho & 0,348 & 0,410 & $-0,615$ & $-0,433$ & 0,778 & $-0,884$ & $-0,444$ & 1,000 & \\
\hline & Valor de $p$ & 0,163 & 0,120 & $0,029^{*}$ & 0,106 & $0,004^{*}$ & $<0,001^{*}$ & 0,099 & . & \\
\hline & $\mathrm{N}$ & 10 & 10 & 10 & 10 & 10 & 10 & 10 & 10 & \\
\hline \multirow{3}{*}{ IIF } & Rho & $-0,109$ & $-0,174$ & 0,335 & 0,175 & $-0,418$ & 0,225 & 0,406 & $-0,251$ & 1,000 \\
\hline & Valor de $p$ & 0,382 & 0,316 & 0,172 & 0,315 & 0,114 & 0,266 & 0,122 & 0,242 & . \\
\hline & $\mathrm{N}$ & 10 & 10 & 10 & 10 & 10 & 10 & 10 & 10 & 10 \\
\hline
\end{tabular}

*Valores significativos $(p<0,05)$ - Coeficiente de Correlação de Spearman

Legenda: N = nomeação; PCC-R = porcentagem de consoantes corretas revisado; PDI = Process Density Index; IRS = índice relativo de substituição; IRO = índice relativo de omissão; IRD = índice relativo de distorção; IIF = índice de inconsistência de fala

de imitação de palavras e entre o IRO e PCC-R, na prova de imitação de palavras. Houve tendência à correlação positiva no G2 apenas entre o IRD da nomeação e PCC-R da imitação. A tendência à correlação negativa ocorreu entre o IRS da nomeação e o PCC-R da imitação, IRO da nomeação e PCC-R da imitação, IRS da imitação e PCC-R da nomeação, IRS e PCC-R da imitação e entre o IRD e IRO da imitação.

\section{DISCUSSÃO}

Os índices de gravidade de fala PCC-R e PDI aplicados às provas do teste de fonologia, em conjunto com a análise do IIF, foram eficientes para diferenciar crianças com TF, com e sem a presença dos processos fonológicos de EF e EP.

Os dados que mostraram que o G1, grupo de crianças sem EP e EF, apresentou maior número de consoantes corretas (medido pelo PCC-R) e menor ocorrência de PF (medido pelo $\mathrm{PDI}$ ), quando comparado ao G2, grupo com EP e EF, evidenciam que os casos de crianças com TF, com a presença de EP e EF acima de 25\%, são mais graves do que os de crianças que não apresentam esses processos fonológicos. A presença de EP e EF no português brasileiro é muito relatada em pesquisas ${ }^{(21-23)}$ e parece estar relacionada à produção $\mathrm{e}$ manutenção do vozeamento pelas pregas vocais ${ }^{(22,24)}$. Esses dois processos (EP e EF) envolvem seis sons vozeados (/b,d,g,v,z,Z/), que, quando produzidos com pouco ou sem vozeamento, resultam em grande comprometimento da análise da fala pela oitiva.

Os índices relativos que demonstraram que, em ambos os grupos, o tipo de erro mais ocorrente foi o de substituição (IRS), foram confirmados pelo ranqueamento intragrupo realizado nas duas provas de fonologia. Um estudo recente ${ }^{(8)}$ mostrou que os índices absolutos e relativos identificaram que o tipo de erro de fala mais ocorrente nas crianças com TF, com dificuldade de base cognitivo-linguística, é a substituição.

O IRS foi maior para o G2 do que para o G1 nas duas provas e, para o IRD, houve tendência à significância na prova de nomeação, com maior ocorrência de distorção no G1. Sabe-se que o tipo de erro e sua ocorrência influenciam a gravidade do $\mathrm{TF}^{(11)}$. Quanto maior a gravidade do $\mathrm{TF}$, mais 
comprometida a inteligibilidade de fala em crianças que apresentam substituições e omissões, em comparação com aquelas que apresentam distorções ${ }^{(8)}$. Como não houve diferença entre as idades dos sujeitos dos dois grupos, o fato de haver tendência à maior ocorrência de distorções no G1 (Tabela 1), não pode ser atribuída às características maturacionais ${ }^{(25)}$, mas sim às relativas aos erros residuais ${ }^{(1,14,26)}$. Esses dados sugerem que a presença do processo fonológico de ensurdecimento possa estar associada à dificuldade no conhecimento da regra fonológica ${ }^{(27) .}$

Outro resultado interessante, diz respeito à inconsistência de fala. A literatura ${ }^{(28)}$ mostra que os pacientes com TF que são inconsistentes costumam apresentar maior gravidade de alteração de fala. Os dados deste trabalho apontaram que, no G2, houve maior número de crianças classificadas como inconsistentes, sugerindo um provável comprometimento da programação fonológica, que resulta em uma dificuldade cognitivo-linguística da representação das regras fonológicas da língua ${ }^{(15)}$.

É importante destacar que a comparação entre as provas de imitação e de nomeação não indicou diferenças para os índices estudados, o que demonstra a efetividade da aplicação desses índices em qualquer amostra de fala ${ }^{(29)}$. As correlações entre os índices, em ambas as provas, também sugerem que esses indicadores mantiveram uma associação de valores nas duas provas. A correlação positiva observada no G1, entre os índices PCC-R e IRD, em ambas as provas, indica que quanto maior o número de produções corretas e de distorções, menor será o número de substituições e omissões ${ }^{(8)}$. A correlação negativa verificada no G2, entre o PCC-R e o IRS, também confirma a relação de que, quanto maior o PCC-R, menor será o IRS em ambas as provas.

\section{CONCLUSÃO}

$\mathrm{O}$ estudo realizado indicou que crianças com TF e presença de ensurdecimento de fricativas e/ou plosivas têm maior comprometimento de fala, demonstrado pelos valores mais baixos de PCC-R, além de valores mais altos de IIF, PDI e dos Índices Relativos.

O erro articulatório mais ocorrente para ambos os grupos foi o de substituição, sendo que a distorção ocorreu mais no grupo sem os processos fonológicos de ensurdecimento, que apresentaram maior valor de PCC-R.

Há evidências de que as crianças estudadas que possuem os processos fonológicos de ensurdecimento têm dificuldade na representação fonológica, o que indica a necessidade de escolha do modelo terapêutico mais adequado.

\section{AGRADECIMENTOS}

À Fundação de Amparo à Pesquisa do Estado de São Paulo (FAPESP) pelo apoio concedido para realização desta pesquisa, processo número 2011/17920-0.

\section{REFERÊNCIAS}

1. Shriberg LD, Austin D, Lewis BA, McSweeny JL, Wilson DL. The speech disorders classification system (SDCS): extensions and lifespan reference data. J Speech Lang Hear Res. 1997;40(4):723-40.

2. Peña-Brooks A, Hedge MN. Assessment and treatment of articulation and phonological disorders in children. Austin: Pro Ed; 2000.

3. Wertzner HF. O distúrbio fonológico em crianças falantes do português: descrição e medidas de severidade. [tese] [LivreDocência Junto ao Departamento de Fisioterapia, Fonoaudiologia e Terapia Ocupacional]. São Paulo (SP): Faculdade de Medicina da Universidade de São Paulo; 2002.

4. Ingram D. Phonological disability in children: studies in language disability and a remediation. $2^{\text {nd }}$ ed. London: Edward Arnold; 1976.

5. Wertzner HF, Herrero SF, Ideriha PN, Pires SCF. Classificação do distúrbio fonológico por meio de duas medidas de análise: Porcentagem de Consoantes Corretas (PCC) e índice de ocorrências dos processos (PDI). Pro-Fono 2001;13(1):90-7.

6. Wertzner HF, Oliveira MMF. Semelhanças entre os sujeitos com distúrbio fonológico. Pro-Fono. 2002;14(2):143-52.

7. Wertzner HF, Francisco DT, Pagan-Neves LO. Fatores causais e aplicação de provas complementares relacionadas à gravidade no transtorno fonológico. Rev Soc Bras Fonoaudiol. 2012;17(3):299-303.

8. Wertzner HF, Santos PI, Pagan-Neves LO. Tipos de erros de fala em crianças com transtorno fonológico em função do histórico de otite média. Rev Soc Bras Fonoaudiol. 2012;17(4):422-9.

9. Wertzner HF, Pagan-Neves LO. A efetividade dos testes complementares no acompanhamento da intervenção terapêutica no transtorno fonológico. Rev Soc Bras Fonoaudiol. 2012;17(4):469-75.

10. Dias RF, Mota HB, Mezzomo CL. A consciência fonológica e a consciência do próprio desvio de fala nas diferentes gravidades do desvio fonológico. Rev CEFAC. 2009;11(4):561-70.

11. Shriberg LD, Austin D, Lewis BA, McSweeny JL, Wilson DL. The Percentage of Consonants Correct (PCC) metric: extensions and reliability data. J Speech Lang Hear Res. 1997;40(4):708-22.

12. Edwards ML. Clinical forum: phonological assessment and treatment: in support of phonological processes. Lang Speech Hear Serv Sch. 1992;23(3):233-40.

13. Wertzner HF, Amaro L, Galea DES. Phonological performance measured by speech severity indices compared with correlated factors. São Paulo Med J. 2007;125(6):309-14.

14. Shriberg LD. Four new speech and prosody-voice measures for genetics research and other studies in developmental phonological disorders. J Speech Lang Hear Res. 1993;36(1):105-40.

15. Castro MM, Wertzner HF. Speech Inconsistency Index in Brazilian Portuguese-Speaking Children. Folia Phoniatr Logop. 2011;63(5):237-41.

16. Holm A, Crosbie S, Dodd B. Differentiating normal variability from inconsistency in children's speech: normative data. Int J Lang Commun Disord. 2007;42(4):467-86.

17. Crosbie S, Holm A, Dodd B. Cognitive flexibility in children with and without speech disorder. Child Lang Teach Ther. 2009;25(2):250-70. 
18. Castro MM, Wertzner HF. Articulatory error consistency in Brazilian Portuguese children with Phonological Disorder. In: 27th World Congress of the Internacional Association of Logopedics and Phoniatrics, Copenhagen. Dinamarca; 2007.

19. Wertzner HF. Fonologia. In: Andrade CRF, Befi-Lopes DM, Fernandes FDM, Wertzner HF. ABFW Teste de linguagem infantil nas áreas de fonologia, vocabulário, fluência e pragmática. Carapicuíba: Pró-Fono; 2004.

20. Andrade CRF, Befi-Lopes DM, Fernandes FDM, Wertzner HF. ABFW Teste de linguagem infantil nas áreas de fonologia, vocabulário, fluência e pragmática. Carapicuíba: Pró-Fono; 2004.

21. Papp ACCS, Wertzner HF. O aspecto familial e o transtorno fonológico. Pro-Fono. 2006;18(2):151-60.

22. Gurgueira AL. Estudo acústico do Voice Onset Time (VOT) e da duração da vogal na distinção da sonoridade dos sons plosivos em crianças com transtorno fonológico [tese]. São Paulo (SP): Universidade de São Paulo; 2006.

23. Yavas M, Hernandorena CL, Lamprecht R. Avaliação fonológica da criança. Porto Alegre: Artmed Editora; 2002.
24. Pagan-Neves LO, Wertzner HF. Elettroglotographic study from Brazilian Portugues fricative voiced sounds. In: The São Paulo School of Advanced Studies in Speech Dynamics (SPSASSD); 2010. Jun 07-11. São Paulo; 2010.

25. Smith A. Speech motor development: integrating muscles, movements, and linguistic units. J Commun Disord. 2006;39(5):33149.

26. Shriberg LD, Fourakis M, Hall SD, Karlsson HB, Lohmeier HL, McSweeny JL, et al. Extensions to the Speech Disorders Classification System (SDCS). Clin Ling Phon. 2010;24(10):795824.

27. Wertzner HF, Santos PI, Pagan-Neves LO. Ocorrência de erros fonológicos de acordo com a gravidade em crianças com transtorno fonológico. No prelo. Rev CEFAC. 2013.

28. Broomfield J, Dodd B. The nature of referred subtypes of primary speech disability. Child Lang Teach Ther. 2004;20(2):135-51.

29. Wertzner HF, Papp ACCS, Galea DES. Provas de nomeação e imitação como instrumentos de diagnóstico do transtorno fonológico. Pro-Fono. 2006;18(3):303-12. 